\title{
Classification of Eye Diseases Using Optic Cup Segmentation and Optic Disc Ratio
}

\author{
B.Balaji Naik ${ }^{1}$, R.Mariappan ${ }^{2}$ \\ ${ }^{1,2}$ Department of ECE, SV College of Engineering, Tirupati-517 507 \\ Email: prof.mariappan.r@svcolleges.edu.in
}

\begin{abstract}
The retinal images are widely used in the diagnosis and treatment of various eye diseases such as diabetic retinopathy, cataract and glaucoma. Normally retinal images are classified manually by clinicians trained in time and process resources. Segmentation imaging of the eye retina provides an important role in the calculation the shape and size of the optic disc and the anterior segment and the abnormal growth of any geometry in the eye region. It automatically and precisely calculates the values of location, position and contour area and a structural part of the image required by ophthalmologists. When there is an elevated intra ocular pressure from the normal condition, the subject is affected by glaucoma and in this condition the retinal nerve fiber layer and the optic disc are affected and this leads to progressive loss of vision. This paper proposes a novel method for glaucoma screening by using optic disc \& cup segmentation. But, the segmentation methods suffer from many problems such as the optimization, and initialization inadequate in noisy images results. In this paper, we are tried to locate the most important region of the eye optic disc and optic cup using a morphological operations. The optic disc is the area on the retina optic nerve axons that enter and leave the eye. The proposed work consists pre processing using morphological operations and then segmentation of optic disc and optic cup by using the morphological operations. These operations are minimizing errors detection limit of the optic disc due to blood vessels cross. Then, the cup to disc ratio (CDR) is calculated using these operations. The cup to disc ratio is more than 0.3 then patients are glaucoma otherwise normal. Spatially Weighted Fuzzy C Mean (SWFCM) clustering method is used to segment the optic disc and Superpixel algorithm is used to segment the optic cup. The segmented optic disc and cup are then used to compute the CDR for glaucoma screening. Optic disc and cup segmentation of fundus image is considered for Diabetic Retinopathy Detection and Glucoma detection. The implemented results using MATLAB shows accurate results obtained both for diabetic retinopathy as well as glaucoma.
\end{abstract}

Keywords: Diabetic retinopathy, Glaucoma, Segmentation, Cup to disc ratio

\section{Introduction}

Glaucoma is a ceaseless eye infection in which the optic nerves continuously harmed. It is the second driving reason for visual impairment, and is anticipated to influence around 80 million individuals by 2020 . Movement of the ailment prompts loss of vision, which happens step by step over a drawn out stretch of time. As the side effects just happen when the illness is entirely cutting-edge, glaucoma is known as the noiseless cheat of sight. Glaucoma can't be cured, however its movement can be backed off by treatment. In this manner, identifying glaucoma in time is basic. Be that as it may, numerous glaucoma patients are ignorant of the malady until it has achieved its propelled stage. In Singapore, more than $90 \%$ of patients are unconscious that they have this condition In Australia, around half of individuals with glaucoma are un analyzed.

\section{Existing Methods}

Ravishankar et al. and other authors showed that blood vessels, exudates, micro aneurysms and hemorrhages can be accurately detected in the images using different image processing algorithms, involving morphological operations. These algorithms first detect the major blood vessels and then use the intersection of these to find the approximate location of the optic disk. Detection of the optic disk, fovea and the blood vessels is used for extracting color information for better lesion detection. But the optical disk segmentation algorithm is rather complex, time consuming, and affected the overall efficiency of the system.

\section{Proposed Method}

This paper proposes superpixel classification primarily based disc and cup segmentations for eye disease screening. An identical conception has been used for vessel segmentation.We calculate centre surround statistics from super pixels and unify them with histograms for disc and cup segmentation. We tend to incorporate previous data of the cup by together with location info for cup segmentation. Supported the metameric disc and cup, CDR is computed for eye disease screening. 

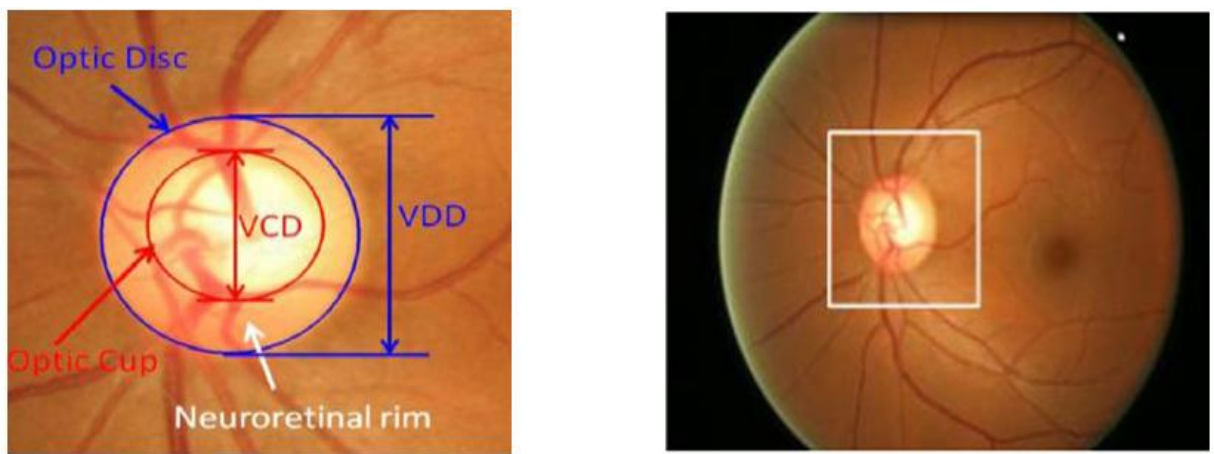

Fig 1 Image of Optic cup and disc

For detection of Diabetic Retinopathy from color complex body part image numerous steps are applied. The varied steps for detection of diabetic retinopathy is shown in Fig. 2. The constituent values of Color complex body part Image square measure for good distorted and also the superior information is employed for analysis of pictures. This suppresses unsought data and enhances needed options. Pre process in brightness correction, edge detection, intensity adjustment, bar chart effort etc.

\subsection{Pre Processing}

For the area of periods of glaucoma the Color Fundus Images are considered as an information. These photos are just the shading pictures which gives the experiences about retina of eye. These photos are preprocessed to improve the way of picture and this preprocessed picture is used for the further stages.

\subsubsection{Morphological Transformation}

Pre-processing is applied for detection of Dark lesions that have dire low content within the inexperienced color plane. Furthermore the background intensity variation of the image within the inexperienced plane is a smaller amount. Therefore the inexperienced channel of a picture is obtained initially and for each component pixel within the image, a region is taken into account focused on the chosen component. If the grey level of the component is lesser than the fraction of the mean of the component is taken into account as darker than the encompassing pixels. The darker area units than the conventional issue layer are detected. Finally a binary image is obtained with non zero values for selected dark pixels. With in the technique planned by Meindert Niemeijer et.al the inexperienced plane of the image. Under goes shade correction. The shade corrected image is operated reworked to section the vessels gift within the image.

In order to eliminate the blood vessels a vascular tree algorithmic rule is planned. The vascular tree is taken into account because the solely a part of the image that's uniform throughout.

Morphological gap with a linear structuring part of size fifteen pixels is employed to get rid of rounded bright zones of size below fifteen pixels. The distinction is improved by getting the total of the highest hat transforms. The ensuing pictures area unit streaky and Gaussian smoothing is employed to eliminate the noise within the image. To fully eliminate dark lesions, a linear gap by reconstruction of size fifteen, a linear closing by reconstruction of size fifteen, and linear gap of size twenty nine is finished or else. The reconstructed image is ablated from the shade corrected image; matched filter of size $11 \times 11$ pixels is then used. A threshold price is ready which ends up in an exceedingly binary image. The binary image isn't a reproduction of the first image as a result region growing is finished exploitation the darkest component because the place to begin. Akara Sopharak et al in their work to discover exudates created use of morphological filtering. The pre-processing of image involves the conversion in RGB to Hyper spectral imaging (HSI) color image, because the intensity elements area unit aloof from the remaining color elements. Distinction reconciling bar graph leveling is applied to tiny regions of the image. The small regions area unit combined by linear interpolation when leveling. Exudates area unit regions of high intensity values .pixels at the structure marked as one and pixels within the background marked as zero. 


\section{Design flow for detection of Diabetic Retinopathy}

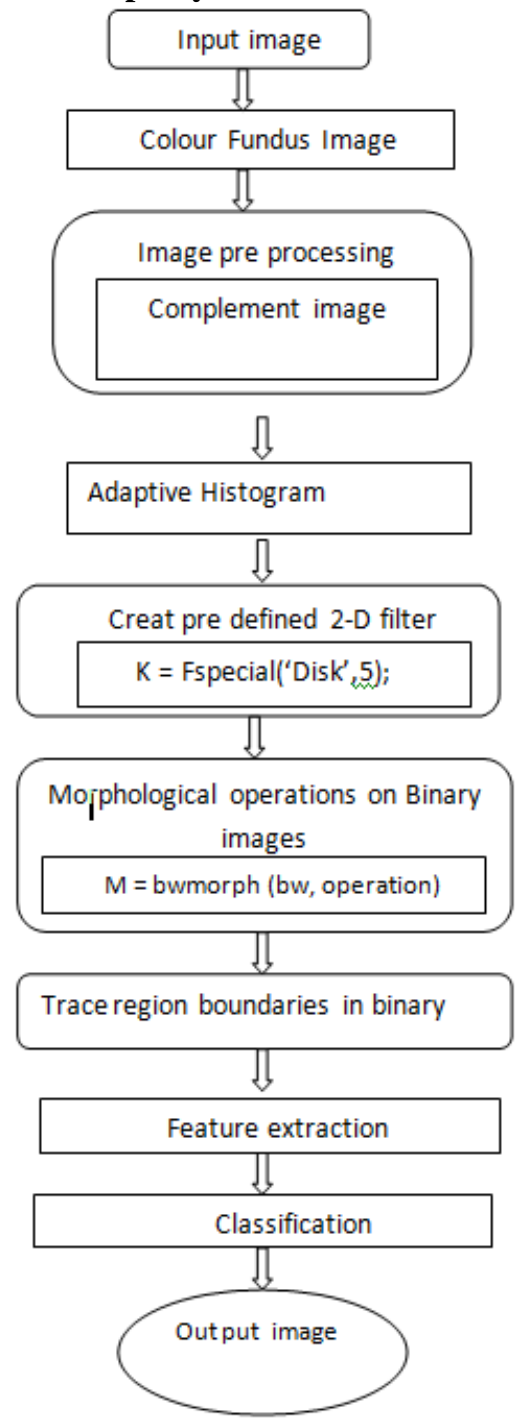

Figure .2: steps for detection of Diabetic Retinopathy

\subsection{Small Aneurysms Detection}

For the effective detection of Diabetic Retinopathy in Diabetic patients Microaneurysms (MA) area unit detected as these area unit the earliest sign of the Diabetic Retinopathy.

\subsubsection{Double Ring Filter}

After image pre-processing, candidate regions for microaneurysms were detected employing a doublering filter. The diameters of five and thirteen pixels for inner and outer ring severally is employed for detection small aneurysms within the encompassing retinal regions of the image. Any potential false positives situated within the regions appreciate blood vessels were removed by automatic extraction of blood vessels from the pictures. 100 twenty six image options were determined and twenty eight elements were hand-picked by mistreatment principal part analysis. The candidate lesions were classified into microaneurysms or false positives mistreatment the rule-based technique and a synthetic neural network.

\subsubsection{Bar Graph Equalisation}

A neural network based mostly preprocessing stage for bar graph equalisation and specialization is used. A window technique uses the regions coated by the window function the input options. For preprocessing, the inexperienced plane shaded color pictures are thought of. If the exudates are the middle picture element then it's thought of as positive sample else it's thought of as negative sample. To find the exudates, a 9x9 sized window is employed. A target vector is formed supported the positive and negative samples and therefore the vector is ready as zero or one. 


\subsubsection{Morphological Filtering}

Morphological closing ends up in twisted vessel like patterns being treated as MA, a perfect operator with all structuring components having giant diameter is employed The binary options, grey level and color options are extracted for the detection of mass.

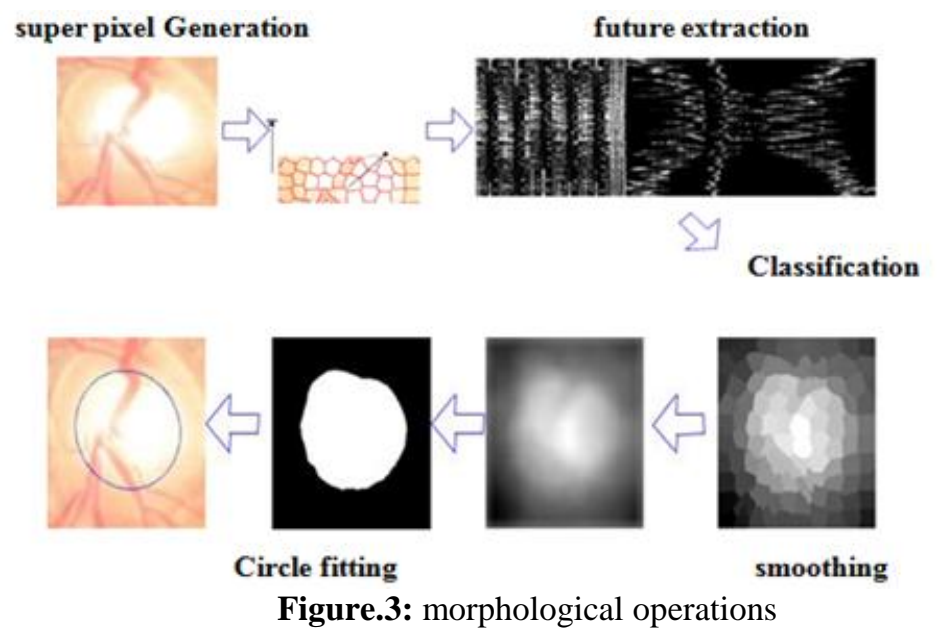

\subsubsection{Density perform}

The spatial relationship of the pixels has thought of to differentiate between the candidate lesions, noise and pigment variability. A density perform is outlined to search out the quantity of comparable picture elements within the neighborhood of the chosen pixel. A threshold price is ready to eliminate pixels happiness to the blind spot and therefore the blood vessels as a result the dark lesions are known.

\subsubsection{Region Growing}

Region growing rule is employed to search out the morphology of the candidate. The options are outlined to more improve the accuracy of detection within the technique. A modification is created so the measures were increased with a multiplier factor for higher discrimination between small aneurysms and alternative components of the vasculature.

\subsubsection{Thresholding}

S.Kavitha et al planned a technique for detection of exhausting and soft exudates whereby the preprocessing involves color area conversion into science lab color area and detection of body structure region. And the science lab color area image is regenerate into binary image by thresholding. The binary image is morphologically closed and a body structure mask is made with Thresholding.

\subsubsection{Native Rotating Crosswise Profile}

Microaneurysm detection through the analysis of directional cross-section profiles is planned by Istvan diseased person et al, focused on the native most pixels of the preprocessed image. The height detection is applied on every profile then a collection of attributes relating to the scale, height, and form of the peak area unit calculated. The applied math measures of those attribute values, because the orientation of the crosswise changes; represent the feature set that's accustomed exclude spurious candidates. 


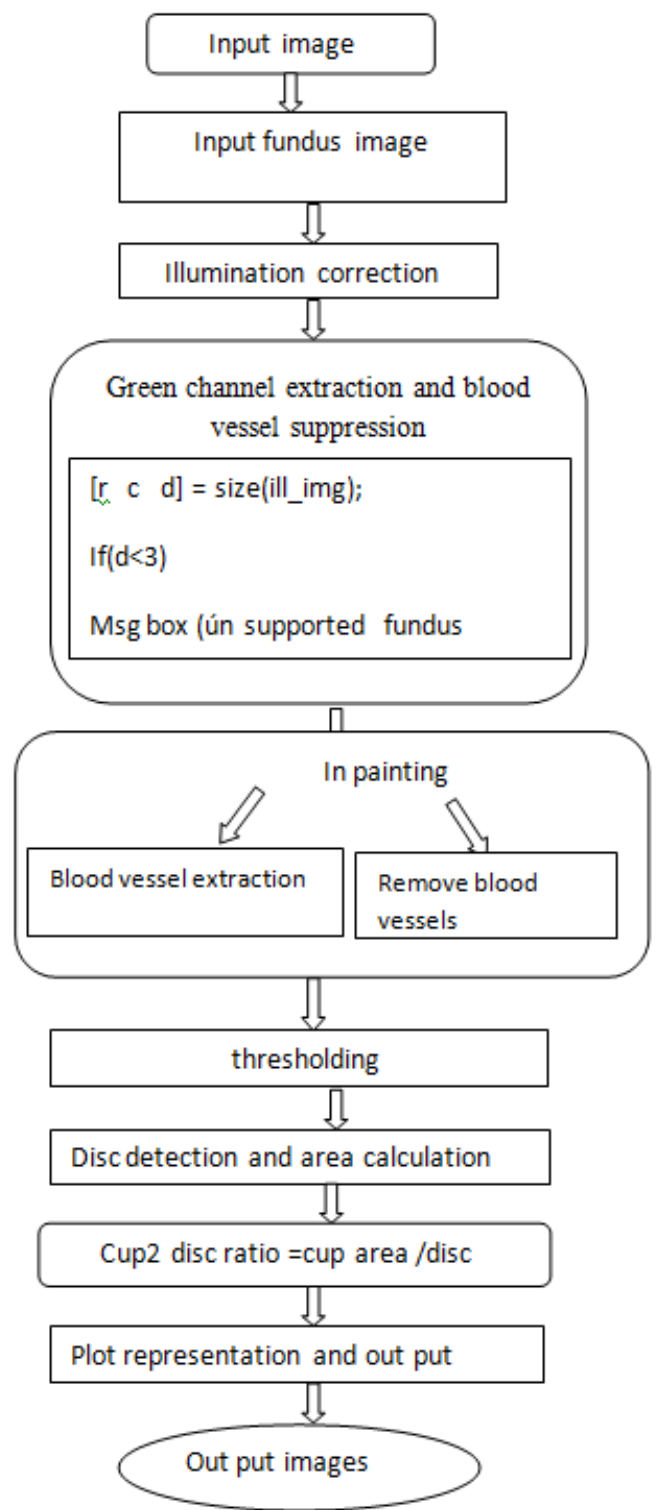

Figure.4: steps for glaucoma detection

\subsection{Exudates Detection}

Exudates area unit any fluid that filters from cardiovascular system of eye into lesions or areas of inflammation.

\subsubsection{Fuzzy C-Means agglomeration}

Fuzzy C-means agglomeration is employed to discover exudates supported four options and eight clusters area unit fashioned for forty retinal pictures. The first image with the exudates region absent is then deducted from the first image to seek out the area units wherever exudates are gift. The cluster image is employed as marker and original image as mask and morphological reconstruction by dilation is applied. Then thresholding is applied and distinction between the first and reconstructed image is found. The ensuing image is superimposed on the first retinal image. Akara Sopharak et al used fuzzy c-means agglomeration for the detection of exudates in tissue layer pictures. Within the pre-processing stage the image the exudates area unit distinguished by means that of the strength. The image is regenerate from the RGB color area to Hyper Spectral Image (HSI) color area and median filtering is applied to the I plane of the image and a distinction increased adaptive bar graph exploit is applied. The quality deviation of the distinction increased image is employed jointly of the options for detective work the exudates. The hue of the HSI image is that the next feature to be extracted. The quantity of edge pixels is that the final feature for agglomeration. The optic disk is removed by victimisation Associate in Nursing entropy feature. To make sure the neighboring pixels area unit enclosed, a dilation method is applied employing a disc structuring component .Then the quantity of white pixels within the image area unit found. 


\subsubsection{K -means clustering}

$\mathrm{K}$ mean clustering is employed to discover exudates and non exudates as a result the amount of clusters shaped. Exudates are placed in high intensity variation where as the opposite lesions are gift within the low intensity variation. Most and minimum intensity values live calculated and function a distance measure. The method is recurrent and therefore the cluster centers are updated in every step.

\subsubsection{Contextual Clustering}

The discourse data is employed to reinforce the detection rate of exudates. Fuzzy art neural network is additionally used for the detection of exudates. From the distinction increased image options adore convexoconcave space, solidity and orientation are extracted. The blind spot is eliminated supported the very fact that it's the article with the most important diameter within the image. Classification relies on four options particularly convexo-concave space, convexo-concave image.

\subsubsection{Linear Discriminate Analysis}

The detection technique of onerous exudates supported Fisher's linear discriminate analysis is developed by Clara I.Sanchez et al. Meindert Niemeijer et al in their work on the automated detection of diabetic retinopathy projected a technique for the first detection of exudates, cotton wool spots and drusens.

\subsection{Classifier}

The classification task consists of derivation a general classification rule for unknown candidates from the coaching set. The strategy for the classification ought to be strong against outliers within the coaching set, as a result of it's terribly troublesome to get AN fully reliable ground truth and therefore the distribution of the options. There exist several classifiers for the detection of MA adore support vector machines, k-nearest neighbor $(\mathrm{KNN})$ technique, linear discriminate analysis, Gaussian filter and neural network are used. For the analysis, A Linear discriminate technique was used as a good classification and every one ways that provided higher results were taken into thought. It's found that AD Tree- a tree based mostly classifier, SVM classifier and supply Regression were used and therefore the performance is analyzed. Fifteen options are extracted from a hundred and fifteen,867 positive and negative samples of exudates pixels. Classification is formed exploitation the Naïve Bayes classifier that repeatedly removes the options until the performance of classifier doesn't improve. The antecedently removed options are also thought of and a few options are found by Bayes classifier. By exploitation these options, SVM classifier is trained. For every combine of parameters, tolerance and radial basis perform is decided.

For the classification of exudates, fifteen options are extracted from the image particularly the element intensity, variance, hue, variety of edge pixels, average intensity of pixel's cluster, size of cluster, average intensity of pixels within the neighborhood of pixels, distance between element cluster and blind spot, filter response of half dozen distinction of Gaussian filters. Bayes classifier is employed for the classification. The KNN classifiers, that include assignment the category to a replacement candidate $(\mathrm{X})$ to that the bulk among the K nearest neighbours of $(\mathrm{X})$ belongs. The benefits of KNN classifiers are the strategy becomes strong against outliers and it's statistic (i.e. No assumption concerning the feature distribution should be made) for moderately giant price of $\mathrm{K}$. One downside of KNN classifiers is that every one neighbours have constant weight for the choice, severally of their distance from the candidate to classify. Moreover, if the coaching set is incredibly uneven for the 2 categories significantly for big $\mathrm{K}$ the strategy may well be failing. So as to beat these issues and to fulfill the on top of necessities, the kernel technique is chosen for density estimation, combined with theorem risk decrease. Three stratified perception design, i.e. One input layer, single hidden layer and single output layer neural network is employed. The input consists of 243 neurons, fifty hidden neurons and output consists of one vegetative cell. A scaled conjugate gradient methodology was used. If the neural network classifier is employed then the output of a network is within the vary of zero to one. A neural network classifier with multilayer perceptron is employed. A log-Sigmoid transfer operate within the output layer and linear operate within the output layer is utilized that makes use of back propagation rule.

\section{Results And Discussion}

As mentioned in previous section it's been noted that the varied classifiers were utilized by existing strategies and performance was evaluated. Completely different values of the parameters determined effective detection of Diabetic Retinopathy and glaucoma . 
Results of diabetic retinopathy detection:

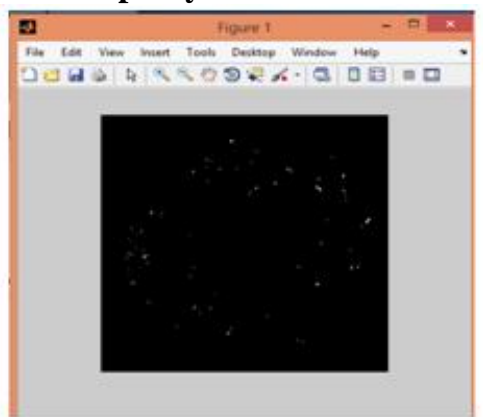

Figure.5: exudates detection

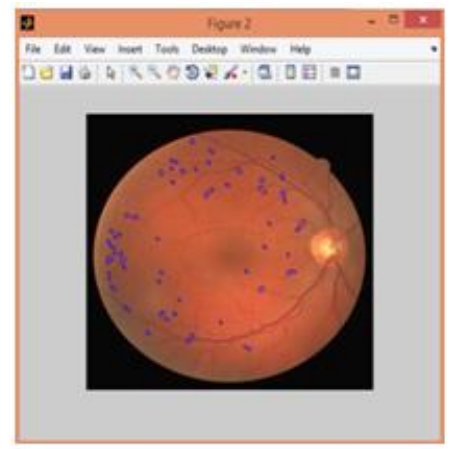

Figure.6: output colour image with exudates

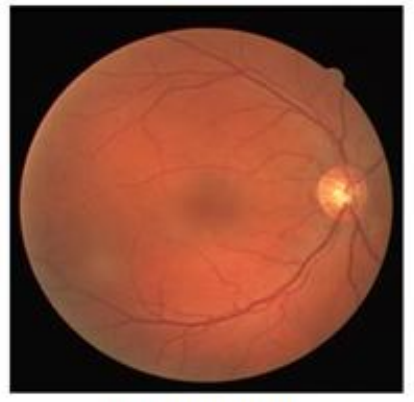

Input funds image

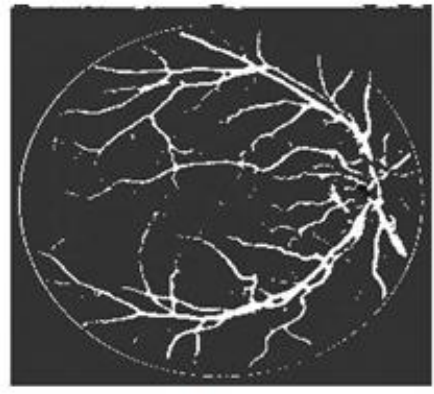

vessel extracted image

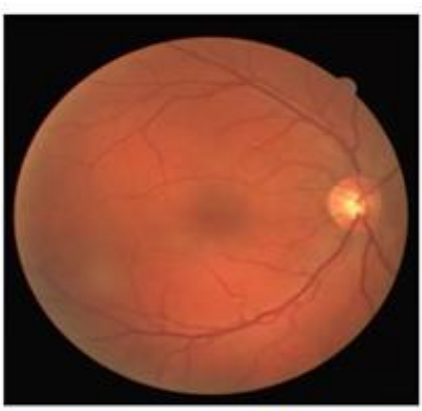

Illumination corrected image

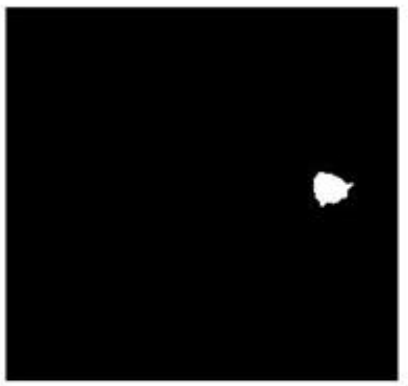

optic cup image

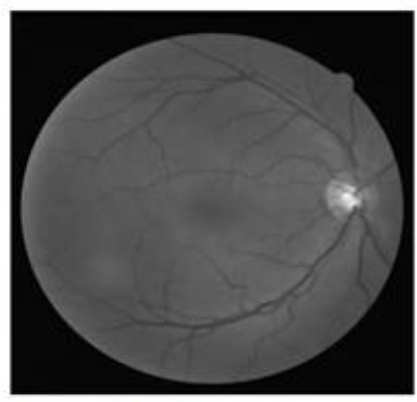

Green channel funds image

Figure.7: Results for glaucoma detection

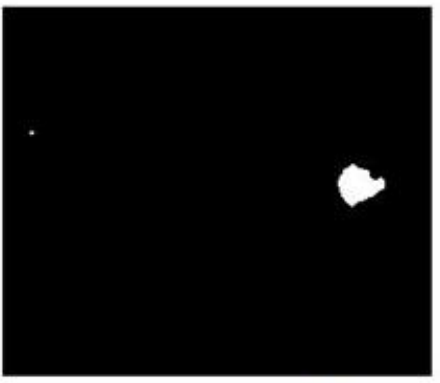

Output disc image

Table I: Cup to disc ratio values

\begin{tabular}{|l|c|l|c|}
\hline \multicolumn{4}{|c|}{ Cup to disc ratio values } \\
\hline Images & normal & glaucoma & diabetic retinopathy \\
\hline Image-1 & 0.3 & 0.472 & 0.472 \\
\hline Image-2 & 0.3 & 0.564 & 0.564 \\
\hline Image-3 & 0.3 & 0.756 & 0.756 \\
\hline
\end{tabular}

\section{Conclusion}

This paper proposes a novel method of classifying eye diseases using optic cup segmentation and cup to disc ratio. After image acquisition, preprocessing is done by applying thresholding, illumination and histogram equalization. The optic disk and cup is segmented using various techniques like Hough transform, kmeans clustering, fuzzy c-means clustering, active contour method, matched filter approach, vessel bends, morphological operations etc. Then cup to disc ration is calculated and classification is done for deciding whether condition of eye is normal or glaucoma based on threshold value of 0.3 . The implemented results using MATLAB shows accurate results obtained both for diabetic retinopathy as well as glaucoma. 


\section{References}

[1]. M Ozaki, Y. Adachi, Y. Iwahori, and N. Ishii, Application of fuzzy theory to writer recognition of Chinese characters, International .Diabetic Retinopathyl, U.S. DEPARTMENT OF HEALTH AND HUMAN SERVICES National Institutes of Health National Eye Institute NIH Publication No: 06-2171Revised 9/03

[2]. Thomas Walter, Jean-Claude Klein, Pascale Massin, and Ali Erginay, - A Contribution of Image Processing to the Diagnosis of Diabetic Retinopathy - Detection of Exudates in Color Fundus Images of the Human Retinall,

[3]. IEEE Transactions On Medical Imaging, Vol. 21, No. 10, October 2002. IEEE Trans. Biomedical Engg, Vol. 51, No. 2, Feb 2004.

[4]. A Novel Approach to Diagnose Diabetes Based on the Fractal Characteristics of Retinal Imagesl, Shu-Chen Cheng and Yueh-Min Huang,IEEE Transactions On Information Technology In Biomedicine, Vol. 7, No. 3, September 2003.

[5]. Automated Feature Extraction in Color Retinal Images by a Model Based Approachll, Huiqi Li and OpasChutatape, IEEE Transactions On Biomedical Engineering, Vol. 51, No. 2, February 2004.

\section{Authors Biography}

Mariappan Ramasamy is Dean of Research \& Development in Sri Venkateswara College of Engg., Tirupati, affiliated to Jawaharlal Nehru Technological University, Andhra Pradesh, INDIA. He is Masters in Electronics \& Communication Engg with specilization in Optical Communication and Doctorate in Information \& Communication Engineering with specilized in Wireless \& Optical Communication Networks. His field of interests includes - Wireless networks, Cognitive networks, sensor networks, Optical networks, Satellite Networks and Network security. He has more than 22 years of Experience in Academic \& Research \& Development Projects. He has published about 48 research papers in reputed Journals and Conference Proceedings. e has been serving as an editorial board member of reputed Journals including IEEE/OSA Journal of Lightwave Technology. He is the Fellow of IE and UWAI. He is also an active member of the Professional Societies: IEEE, CSI, ISTE, ACEEE and IAENG. His current nature of work includes Project development, Researching the new technology development to implement in various research projects in linked with the Industry and Society. He has received ISTE National award for the Best National Projects, Innovation for Entrepreneurship Development Centre (IEDC) Project award, Accenture innovation award, Techknow award, etc. Also he has received research grants from DST, DRDO, CSIR, TNSCST, etc for Research Projects and allied activities.

B.Balaji Naik is a M.Tech (VLSI) Scholar under the Department of Electronics and Communication Engineering, SV College of Engg, Tirupati. His fields of interest includes, VLSI,, Signal Processing and Image Processing algorithms. 